\title{
DISPERSION OF THIRD ORDER OPTICAL NONLINEARITY OF PSEUDOISOCYANINE J-AGGREGATES
}

\author{
R. GADONAS, A. PUGZLYS \\ and K.-H. FELLER ${ }^{\mathrm{b}, *}$ \\ ailnius University, Laser Research Center, Sauletekio ave. 10, LT-2054 \\ Vilnius, Lithuania \\ ${ }^{\mathrm{b}}$ Fachhochschule Jena, Fachbereich Medizintechnik/Physikalische Technik, \\ Tatzendpromenade 1b, D-07745 Jena, Germany
}

(Received 17 April 1996)

\begin{abstract}
Dispersion of degenerate four-wave mixing (DFWM) efficiency near the exciton resonance of aggregates of 1,1'-diethyl-2-2'-cyanine (pseudoisocyanine, PIC) chloride is reported. Correlation with the spectrum of imaginary parts of nonlinearity obtained by pump and probe techniques is discussed. Third order nonlinear susceptibility of PIC aggregates has been calculated supposing highest density packing attainable by Langmuir-Blodgett technique. The nonlinear figure-of-merit of aggregates was evaluated, which is intensity dependent due to exciton annihilation. Observed intensity and wavelength dependence of mixing efficiency is qualitatively explained in terms of absorption saturation, exciton annihilation, and subsequent generation of an excess diagonal disorder by vibrational energy accumulated in aggregates.
\end{abstract}

Keywords: Degenerate four wave mixing; cyanine; J-aggregates; nonlinear susceptibility; dispersion; exciton; annihilation; vibrational energy redistribution; figure-of-merit

\section{INTRODUCTION}

J-aggregates of polymethine dyes have become a focus of interest in the interdisciplinary field of molecular nonlinear optics [1-5] and Langmuir-Blodgett engineering [6-8]. Polymethine dye J-aggregates were formed in LB layers, and storage of optical information in mono-

*Author to whom correspondence should be addressed. 
layers and mixed multilayers has been recently demonstrated $[8,9]$. Sharp and intense absorption bands at room temperature provide the possibility of recording optical information by using the photoablative technique $[6,7]$ or photodissociation $[8,9]$ (photodesordering) of $\mathrm{J}$-aggregates. Although the multicolour information storage devices exploit intense and narrow absorption bands of aggregates, the physical mechanism of storage is mostly thermal change in steady-state spectral characteristics. For fast signal processing based on electronic nonlinear polarisation, the other set of features would be desirable, and these include high photochemical stability, high nonlinear susceptibility, and fast response time, as well as the possibility of phase matching of interacting waves. In the case of polydiacetylenes that show high third order susceptibilities and fast response time, the photostability is a limiting factor for commercial application of this class of nonlinear materials. Fullerenes have recently revealed high susceptibilities and no photodegradation at off-resonance case of DFWM with $1064 \mathrm{~nm}$ picosecond pulses [10]. The $\chi^{(3)}$ values of $(6 \pm 4) \times 10^{-8}$ esu for the solid state of $\mathrm{C}_{60}$ were reported by Blau et al. [11]. Although a $\chi^{(3)}$ value for $1064 \mathrm{~nm}$ of $3.3 \times 10^{-9}$ esu measured in nanosecond DFWM experiments by Gong et al. [12] is considerably lower, it is also among the highest measured susceptibilities having an electronic polarisation character. The nonlinear optical characteristics of fullerenes is still a subject of controversy, and the deduced quantities by different groups scatter within 2-3 orders of magnitude $[10-15]$. Despite the high predicted theoretically $[4,16]$ and experimentally determined third order nonlinear susceptibilities $[1,3,16]$, the capability of nonlinear processing of fast optical signals by the use of J-aggregates has not been studied. Ying Wang [3] used absorption spectroscopy and DFWM with $6 \mathrm{~ns}$ pulses to estimate nonlinear absorption and power dependence of DFWM reflectance in PIC chloride J-aggregates at room temperature. The slope of only 0.7 for log$\log$ plot of power dependence of phase conjugated reflectance was pointed out. Using Kramers-Kronig analysis changes in refraction were obtained. Quantitative data for nonlinear absorption were shown to compare favourably with those for semiconductor-doped glasses and polydiacetylene.

F. Spano et al. $[4,17]$ provided detailed theoretical calculations for phase conjugated DFWM and pump-probe signals in aggregates and 
the scaling of susceptibility with aggregate size. Pure electronic response including contribution of biexciton resonance and superradiance was considered. A. Laubereau and coworkers [18] and T. Kobayashi and coworkers [19] have investigated femtosecond response of pseudoisocyanine J-aggregate nonlinear absorption. Recently F. Sasaki and Sh. Kobayashi [5] reported an anomalous intensity dependence of both absorption changes and DFWM signal for PIC bromide aggregates at $77 \mathrm{~K}$ which was ascribed to coherent exciton-exciton interaction.

On the other hand, the complexity of time and intensity dependence for absorption changes of PIC aggregates are known to be caused by exciton annihilation and subsequent changes in aggregate spectrum due to vibrational excitation [20-23]. Therefore, annihilation as well as secondary vibrational energy dynamics in the aggregate affect the nonlinearity and its time response in DFWM $[1,23]$. The influence of annihilation in decay of DFWM signal was recently observed in case of fullerenes [24]. In the present paper we report further results of DFWM of tuneable picosecond pulses in J-aggregates of PIC in the presence of this complex and strongly intensity dependent relaxation.

\section{EXPERIMENTAL}

DFWM experiments were carried out with tuneable 20 ps pulses of optical parametric oscillator (OPO) pumped by second harmonic of modelocked Nd:YAG laser radiation [25]. The OPO output was frequency doubled in KDP crystal that ensured up to $25 \mu \mathrm{J}$ energy per pulse at wavelengths $560-580 \mathrm{~nm}$. The DFWM experiments with J-aggregates (for a detailed description see also [1]) were similar to those often performed with other nonlinear materials $[10,14]$. The diagram of experiments is shown in Figure 1. The OPO output beam was divided into three beams in such a way that they were approximately equal by energies at the sample cell and had the same polarisation.

The beams 1-3 were crossed in the cell so that 1 and 2 were antiparallel, and the angle between 1 and 3 was $10^{\circ}$. The proper timing of pulses in the cell was obtained by delay lines DL1 and DL2. The cross section of all three beams was about $3 \mathrm{~mm}^{2}$ in diameter at the sample. The phase conjugated beam was reflected by $60 \%$ mirror and pulse energy was measured by a photodiode. Another photodiode 


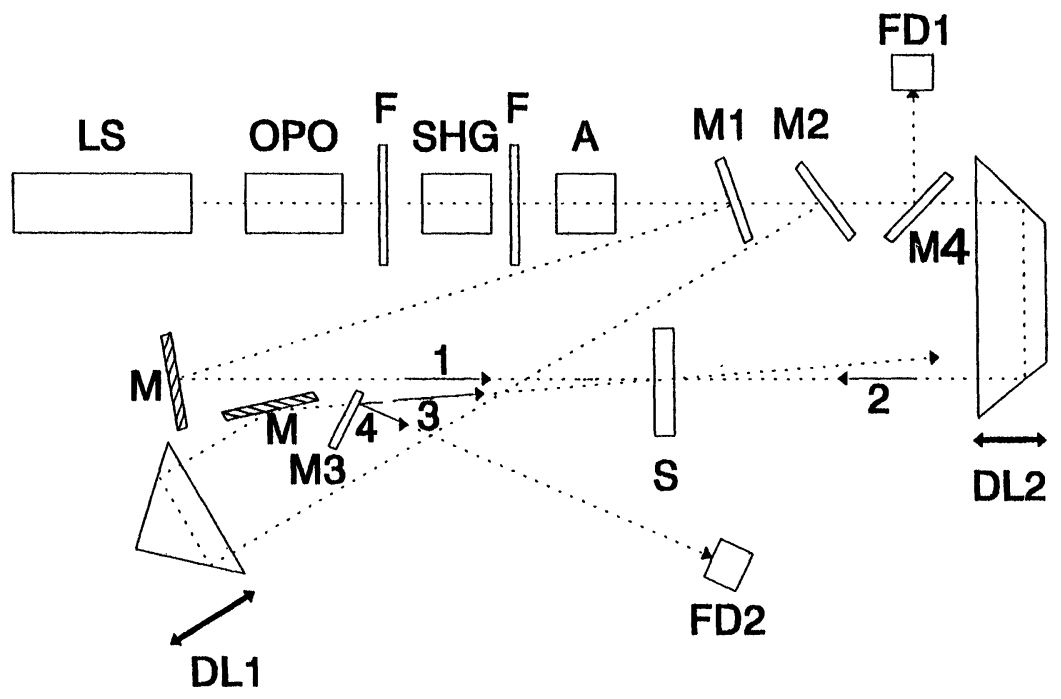

FIGURE 1 Experimental setup. LS-laser and amplifier system, OPO-optical parametric oscillator, F-filter, SHG-second harmonic generator, A-attenuator, M1 …M4-beam splitter, FD-photodiode, M-100\% reflectance mirror, S-sample, DL1, DL2-delay line.

was used to measure the reference signal. The energy of all three beams was changed within more than one order of magnitude by neutral density ink attenuator.

Aggregate samples of 1,1'-diethyl-2,2'-cyanine chloride (pseudoisocyanine, PIC, Filmfabrik Wolfen $\mathrm{GmbH}$ ) were prepared by dissolving $\mathrm{PIC}$ in water at $10^{-3} \mathrm{M}$ concentration and mixing with equal amount of $0.4 \mathrm{M}$ aqueous solution of $\mathrm{NaCl}$. Measurements were carried out using thin $0.12 \mathrm{~mm}$ glass cells. The absorption spectrum of PIC J-aggregates in aqueous solution at concentration of $5 \cdot 10^{-4} \mathrm{M}$ is depicted in Figure 2. The J-aggregate band has its maximum at $573 \mathrm{~nm}$. The width of the narrow band is $60 \mathrm{~cm}^{-1}$ on the red side, whereas the blue wing is inhomogeneous broadened with a width of $160 \mathrm{~cm}^{-1}$.

\section{RESULTS AND DISCUSSION}

Considering steady state absorption of aggregates and parallel polarisation of all interacting beams, it is evident that we have a 


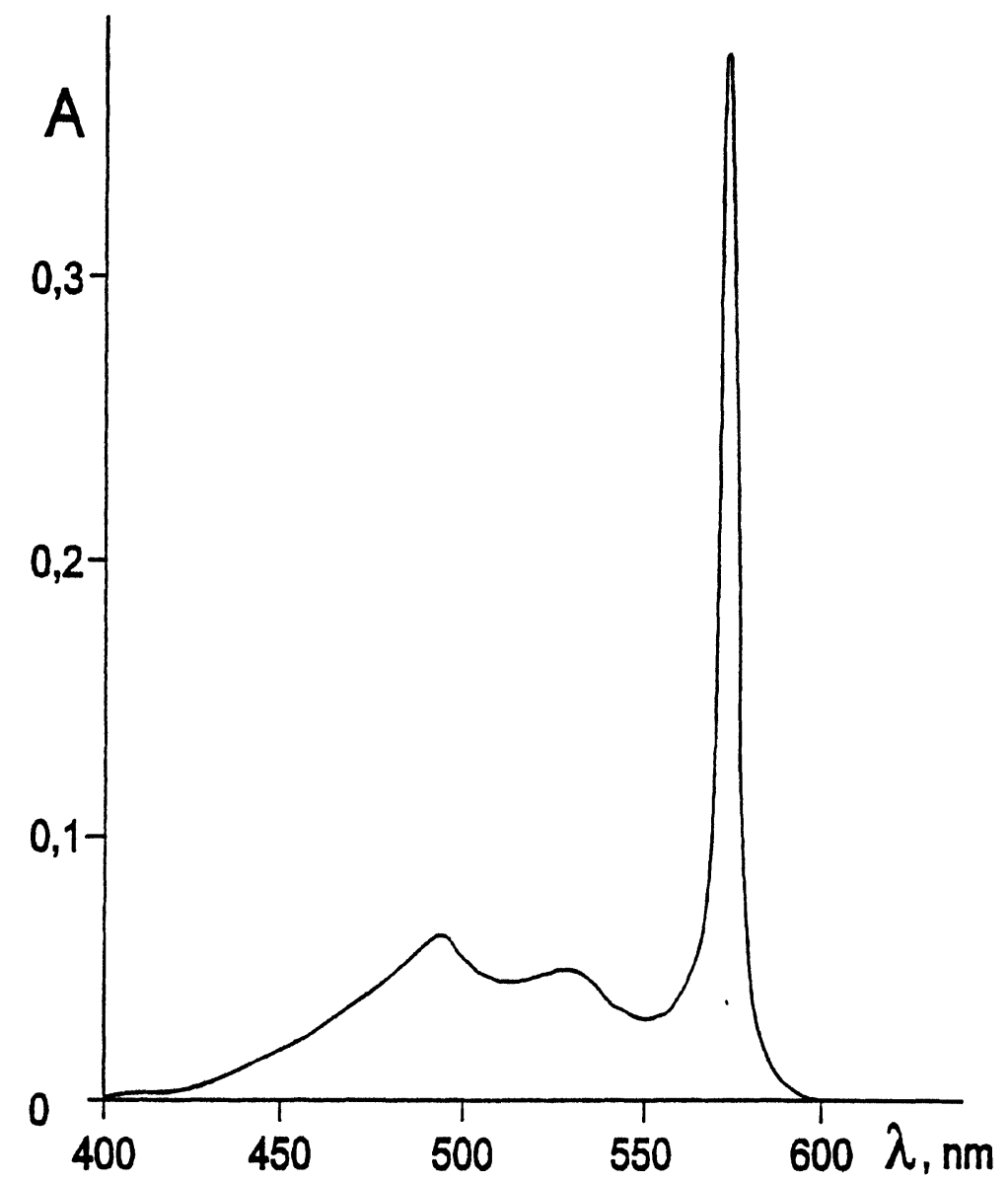

FIGURE 2 Absorption spectrum of PIC in aqueous solution $\left(5 \cdot 10^{-5} \mathrm{M}\right)$ with added concentration of $0.2 \mathrm{M} \mathrm{NaCl}$.

nonlinear optical process governed by spatial modulation of population of real excitations. The phase relaxation time for J-aggregates at room temperature was found to be $70 \mathrm{fs}$ [18], which is short compared to pulse duration. Therefore no significant contribution of coherent interplay between light and matter polarisation is expected. Absorption saturation as well as changes in refractive index due to real excitons with no phase memory cause the process. Moreover, at the incident light intensities used in our experiments the exciton anni- 
hilation plays a significant role in decay of exciton population especially close to resonance $[18,20,21,26]$. Three dimensional diffusion of excitation in the ensemble of aggregates is considered here and the annihilation is written in the form as it follows from the classical binary collision theory. In this theory the annihilation is described by the quantity $\gamma(t)$ which is connected straight with the pair-correlation function $g(r, t)$, i.e., $\gamma(t)=\Sigma_{r} \lambda(r) g(r, t)$, where $\lambda(r)$ is the annihilation probability. This theory is adequate for the description of the annihilation processes in the absence of motion-limited diffusion, whereas a time-dependent function should be used for the one-dimensional exciton diffusion within the aggregate. If the annihilation process is becoming dominant it causes fast accumulation of excess vibrational energy, which in turn is known to strongly influence $\mathbf{J}$-aggregate absorption $[22,23]$. It results in bleaching as well as broadening of $\mathrm{J}$-band which recovers within a time of $18 \mathrm{ps}$ [18]. There has been until now no data on response of refractive index changes to vibrations. Furthermore the transition from the excited state has to be taken into consideration in four wave mixing spectroscopy with femtosecond time resolution [27].

The intensity dependence of phase conjugated signal reveals additional features of interaction. In Figure 3 the intensity of converted signal $I_{\mathrm{DFWM}}$ is depicted vs. the intensity of the three interacting beams at the wavelength corresponding to maximum conversion $\left(\lambda_{\mathrm{exc}}=\right.$ $575 \mathrm{~nm}$ ). The ascent of linear regression in logarithmic scale is 1.5 only instead of 3 expected for non-saturated case (under off-resonance conditions). It shows that annihilation and secondary processes contribute to nonlinearity at $574 \mathrm{~nm}$ resulting in decrease of the ascent. Qualitatively, the role of annihilation can be taken into consideration in the following way. Suppose the signal is due to diffraction of beam 3 on the transmittance grating formed by beams 1 and 2 and originates from population of excited states in aggregate (see Fig. 1). When relatively long $20 \mathrm{ps}$ pulses of megawatt power are applied, the annihilation results in an equilibrium exciton population density that can be estimated using Paillotin's approach [28].

$$
\frac{d n}{d t}=\sigma(1-n) I-k n-\frac{\gamma}{2} n^{2} N
$$




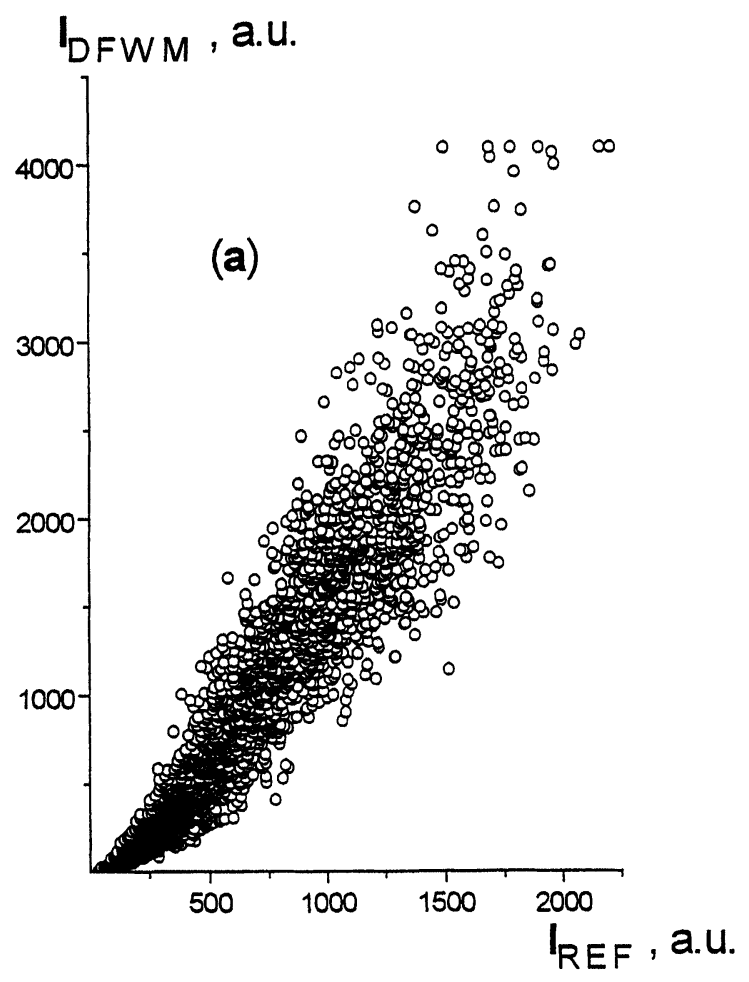

FIGURE 3 Dependence of DFWM signal in arbitrary units in a) linear scale b) logarithmic scale upon the reference intensity which is proportional to the intensity of the interacting beams at wavelength $575 \mathrm{~nm}$ (in exact resonance). In Figure $3 \mathrm{~b}$ the linear regression with a correlation coefficient of 0.93 is drawn into the experimental points.

Here $n$ is dimensionless exciton population (volume density normalised to monomer concentration), $I$ is incident light intensity in photons $/ \mathrm{cm}^{2}, \sigma$ is absorption cross section in $\mathrm{cm}^{2}, k$ is annihilationfree decay rate in $\mathrm{s}^{-1}, \gamma$ is annihilation constant in $\mathrm{cm}^{3} / \mathrm{s}$, and $N$ is monomer concentration in $\mathrm{cm}^{-3}$.

In our intensity range at $574 \mathrm{~nm}$ excitation the annihilation is dominating path of relaxation $[20,23]$. It is evidently considering that at incident light intensity $100 \mathrm{MW} / \mathrm{cm}^{2}$ corresponding to $2.6 \times 10^{15}$ photons $/ \mathrm{cm}^{2}$ per 20 ps pulse the fluorescence quantum yield is almost totally quenched and the decay is not resolvable even with 4 ps pulses [20]. Therefore, within the pulse the population density is limited due to nonlinear increase in decay rate caused by annihilation. The 


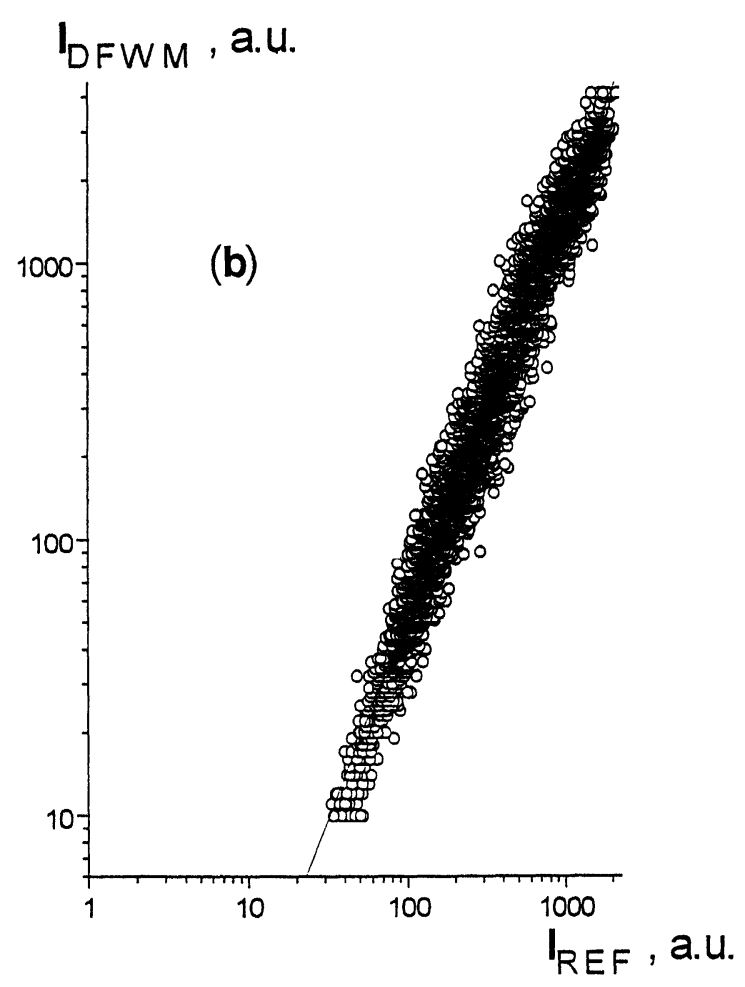

FIGURE 3 (Continued)

simplest estimation on the values of equilibrium population may be obtained as the stationary solution for Paillotin's equation. Neglecting annihilation-free decay term in Equation (1), which is slow compared to exciton generation and annihilation, it gives

$$
n_{\max } \cong \frac{1000}{c N_{A}} \sqrt{\frac{2 I(1-T)}{\gamma \cdot L \cdot h v}}
$$

Here $c$ is molar concentration of dye, $N_{A}$ is Avogadro number, $I$ is intensity in $\mathrm{W} / \mathrm{cm}^{2}, T$ is sample transmittance, $L$ is cell length in $\mathrm{cm}$, and $h v$ is the photon energy in $\mathrm{J}$. Not surprisingly at $100 \mathrm{MW} / \mathrm{cm}^{2}$ we estimate $n_{\max }=0.015$, which corresponds to 1 excitation per 60 monomer molecules. The square root dependence of $n_{\max }$ on $I$ is a key to qualitative explanation of the decrease in power $P$. The decrease in 
amplitude of transmittance grating compared to situation $n \sim I$ at no annihilation and no population saturation results in decrease in ascent. Although the saturating intensity for exciton population defined as

$$
I_{\mathrm{sat}}=\frac{k \cdot h v}{\sigma}
$$

gives the value of about $2 \mathrm{MW} / \mathrm{cm}^{2}$, at this power the integral number of photons absorbed in $20 \mathrm{ps}$ pulse results in population less than 0.04 , that is the pulses are too short to reach saturation. On the other hand, at $I_{\text {sat }}$ the annihilation limits the population at the level of 0.002 , that is far below saturation. Thus, in aggregates due to exciton annihilation no saturation of exciton population occurs [23]. For the complete wavelength-dependent analysis, effects such as electron-phonon coupling as well as transitions from $n$-exciton states to $(n+1)$-exciton states $(n>1)$ should be taken into account.

In our DFWM experiments the backward reflected phase conjugated signal was observed in the range $565-581 \mathrm{~nm}$, and its magnitude as well as intensity dependence were found to be strongly wavelength dependent. The wavelength dependence of conversion efficiency defined as

$$
\varepsilon_{\mathrm{eff}}=\frac{I_{\mathrm{DFWM}}}{I_{1}+I_{2}+I_{3}}
$$

is shown in Figure 4. The measured efficiency values range from $10^{-6}$ to $4 \cdot 10^{-5}$ at $100 \mathrm{MW} / \mathrm{cm}^{2}$ peak power of incident interacting pulses. For comparison, the spectrum of aggregate absorption changes together with that of the change in the refraction index are depicted in Figures $5 \mathrm{a}$ and $5 \mathrm{~b}$. The curves in Figure 5 were measured at different conditions (pulse duration, intensity, etc.) therefore it is difficult to use them to deduce quantitative characteristics. They are depicted to support the qualitative explanation of the measured data.

It is important to note that the maximum efficiency observed at $575 \mathrm{~nm}$ is slightly red shifted compared to the absorption maximum $(573 \mathrm{~nm})$. It is in rather good correlation with the electronic part of light induced absorption changes (low intensity with no annihilation 


$$
\varepsilon_{\text {eff }} \times 10^{-5}
$$

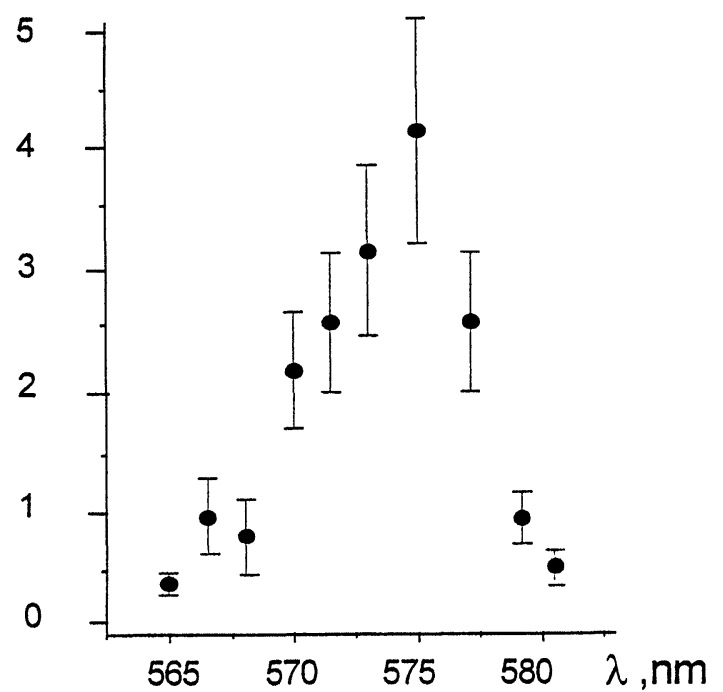

FIGURE 4 Wavelength dependence of conversion efficiency $\varepsilon_{\text {eff }}$ in PIC J-aggregates at excitation intensity $I_{\text {exc }}=100 \mathrm{MW} / \mathrm{cm}^{2}$ peak power.

and vibrational contribution) shown in Figure 5a. The latter was ascribed to the bleaching of single exciton transition [31] (region $\lambda>573 \mathrm{~nm}$ in Figure 5a) together with transient absorption (region $\lambda<573 \mathrm{~nm}$ ) related to transition to two-exciton state $[19,30,31]$. The single exciton bleaching reaches the highest value at $575 \mathrm{~nm}$, which results in high mixing efficiency via transmittance grating. Not only the position of the maximum but also good correlation of the dispersion curve of mixing efficiency with that of bleaching in the long wavelength wing of $J$ band $(\lambda>573 \mathrm{~nm})$. Nevertheless, it does not allow us to draw conclusion about dominant role of absorption saturation in this range. It is difficult to evaluate the role of nonlinear refraction although its contribution is also present at least at $\lambda>575 \mathrm{~nm}$. We do not know what is the result of the interference of amplitude and phase grating in this region. The related shape of dispersion curve corresponding to pure electronic component of transient spectrum is not known. Data of Yink Wang [3] calculated by Kramers Kronig analysis and reproduced in Figure $5 \mathrm{~b}$ correspond to 


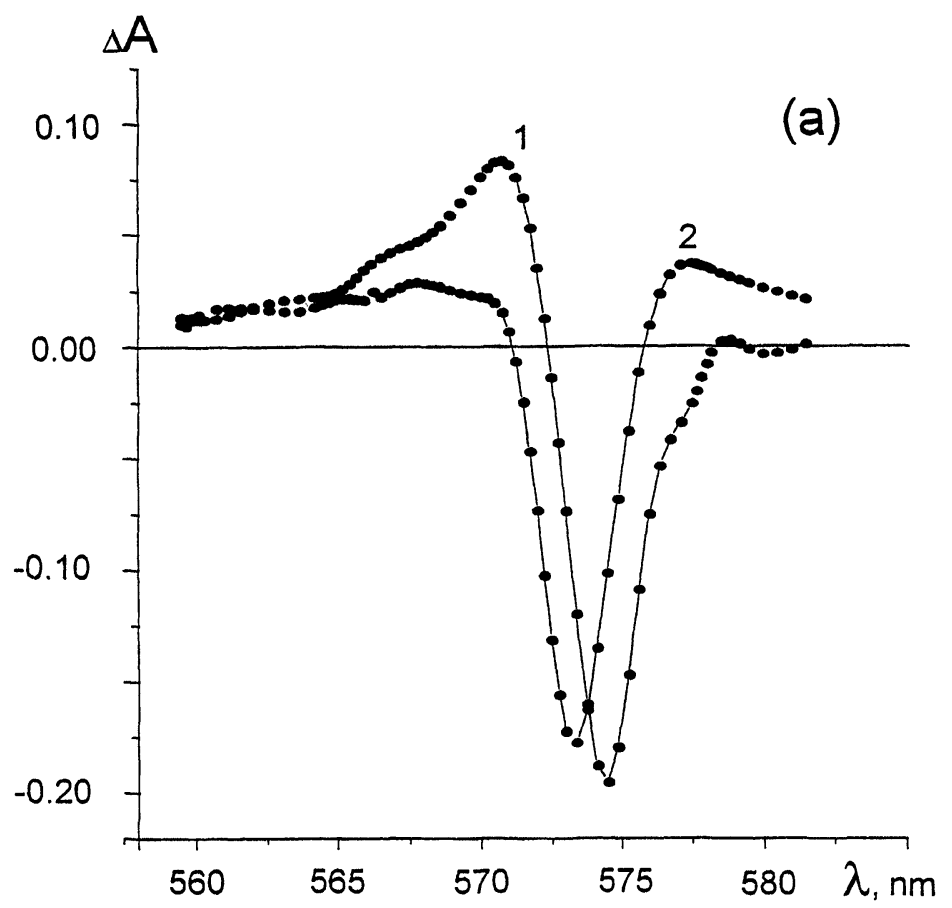

FIGURE 5 a) Spectrum of PIC-J-aggregate absorption changes together with the b) spectral dependence of the change in the index of refraction $(\Delta n)$ obtained through a Kramers-Kronig analysis (the latter was taken from [20]).

a different case where vibrational contribution is obvious, the spectrum of absorption changes has a character of homogeneous bleaching corresponding in reasonable extent to vibrationally "hot" aggregate (see discussion below).

The contribution of transient absorption with maximum at $565 \mathrm{~nm}$ to conversion efficiency seems to be considerably weaker. Although no absorption changes due to exciton population appear at $573 \mathrm{~nm}$, the measured FWM efficiency is high enough. This is due to transmittance modulation caused by population of vibrational degrees of freedom and aggregate dissociation (disordering) by excess vibrations. The latter phenomena may be characterised as the loss of coherent link between monomers in aggregate due to population of such vibrational degree of freedom as internal rotation made of individual 


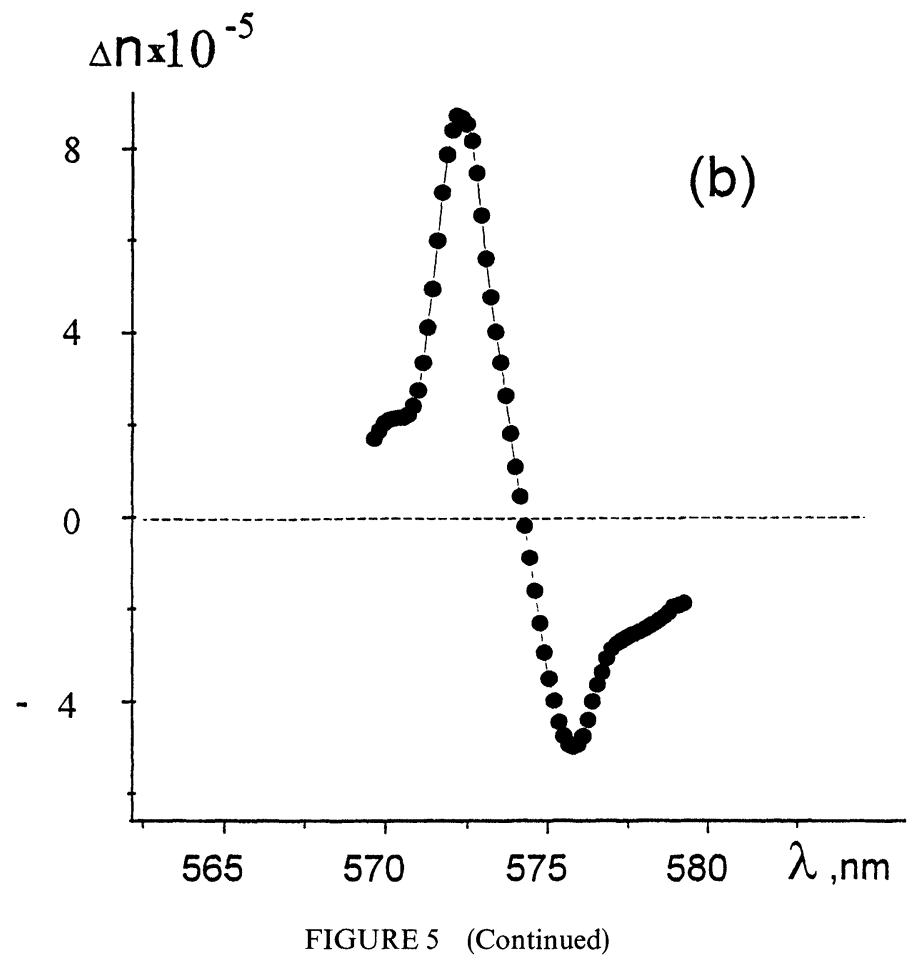

monomers. At intensities used in our experiments, it results in bleaching of J-band with maximum absorption changes at $573 \mathrm{~nm}$ [1]. The transient spectrum with the contribution of vibrationally excited aggregate species is shown in Figure 5a. It can explain the efficient mixing around $573 \mathrm{~nm}$ as caused by nonlinearity originating from secondary vibrational excitation.

Due to limited intensity range of our experiments, which is caused by irreversible changes of aggregate properties at high energy side and the scattering noise at low energy side, the same simple and rough analysis of power dependences of signal was used also within J-band. The correlation coefficient of linear regression in logarithmic scale within the whole studied wavelength range was above 0.85 . Thus, to show variation in intensity dependence of signal with wavelength, the power parameter $P$ was introduced supposing

$$
I_{\mathrm{DFWM}} \propto\left(I_{\mathrm{Ref}}\right)^{P}
$$


where $I_{\text {Ref }}$ is the reference signal. Although this assumption has no physical reasons in resonance, where the dependences deviate from exponent, the $P$ parameter can be regarded as an overall measure of sensitivity of the signal to intensity variations of three interacting beams. The dispersion of $P$ is shown in Figure 6. The overall power is strongly dependent upon the resonance condition. Whereas at the wings of the $\mathrm{J}$-aggregate it is close to 3 , in the exact resonance $(573 \mathrm{~nm})$ the value is 1.25 , and 1.5 in case of maximum conversion $(575 \mathrm{~nm})$. As can be seen from the error bars in Figure 6, with the exception of the wings the intensity dependence is not adequately described by a simple power law. The deviations from single exponent especially in resonance conditions, as discussed above, are due to higher exciton population densities, and contribution to mixing of annihilation and secondary effects $[1,27]$. To calculate nonlinear susceptibility the adequate physical model is necessary. In resonance case it is difficult without full quantitative characterisation of changes in absorption and refractive index related to both electronic and vibrational excitations as well as their time behaviour. Therefore the simple approach often used in off-resonance nonlinear optics where material response is simplified to third order susceptibility was applied in almost off-resonance case in the long wavelength J-band edge. In the latter case the third order intensity dependence of reflected signal was experimentally found [1].

In this case we used a simple relationship given in [4] to estimate near-resonance $\chi^{(3)}$.

$$
I_{4}=\frac{4 \pi^{4} \mu_{0}}{n^{4} \lambda^{2} \varepsilon_{0}^{3}}\left(\chi_{\mathrm{MKS}}^{(3)}\right)^{2} L^{2} I_{1} I_{2} I_{3}
$$

Taking into account

$$
\chi_{\text {esu }}^{(3)}=8.1 \cdot 10^{18} \chi_{\mathrm{MKS}}^{(3)}
$$

we calculated for the third order susceptibility at $579 \mathrm{~nm}$ a value of $1.2 \cdot 10^{11}$ esu. Although this value of $\chi^{(3)}$ describes adequately the power dependence of DFWM signal using formulae for off-resonance case, it should be regarded only as an oversimplified characteristic of aggregate as a nonlinear medium. In other words, it means suscepti- 


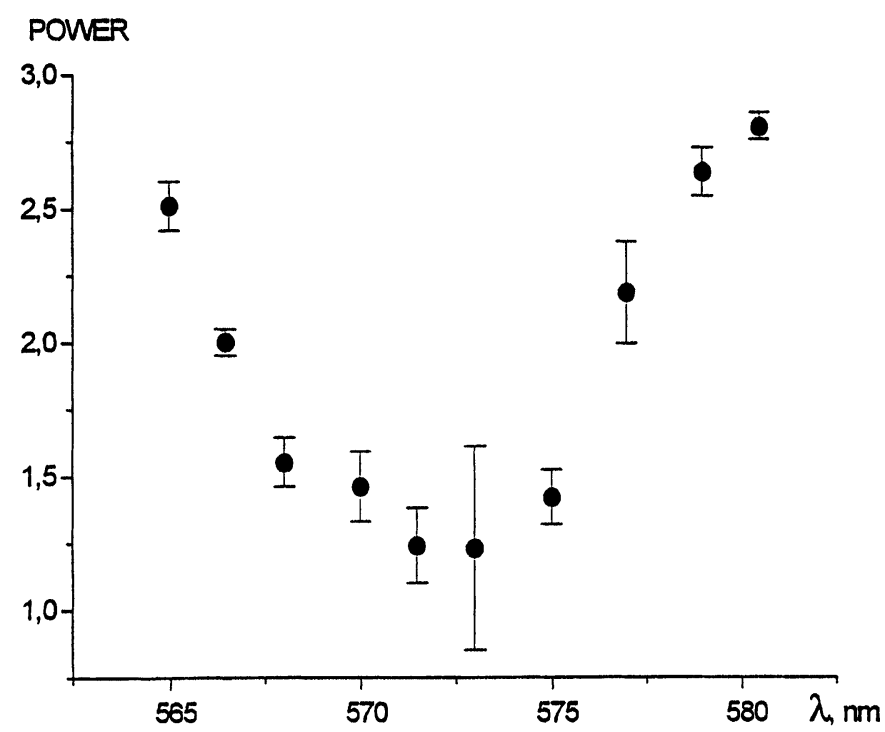

FIGURE 6 Power $P$ dependency of converted signal intensity on the intensity of the interacting beams (here called the reference intensity due to the fact that the three interacting pulses have nearly the same intensity for highest DFWM efficiency).

bility of an off-resonance nonlinear medium that would ensure the measured phase conjugated reflectivity or energy exchange. The resonance situation is much more complicated as it was discussed. Nevertheless, the above evaluations make possible comparison of aggregates and other nonlinear media.

To compare the third order nonlinear constants of different materials is not so simple. Apart from susceptibility, nonlinear figure-of-merit is introduced as an important parameter of a medium. There are different definitions of figure-of-merit. The choice of an adequate definition depends on the nonlinear process considered and the character of material response to light. With respect to the latter, figures-of-merit can be divided into those for resonance and off-resonance cases. For the resonance DFWM process involving real excitation, free excitons and impurities, interband transitions in semiconductors, absorption saturation in organic compounds, the so-called general figure-of-merit

$$
F M=\frac{\chi^{(3)}}{\alpha \cdot \tau}
$$


is used [32]. Here $\alpha$ is the (linear) absorption coefficient in $\mathrm{cm}^{-1}$, and $\tau$ is the lifetime of elementary excitation induced by the resonant transition that contributes to $\chi^{(3)}$. Thus considering the upper limit for response time being annihilation-free lifetime of $\mathrm{J}$-aggregates ( $400 \mathrm{ps}$ [20]), and the lower limit at strong annihilation case resulting in nearly pulse-limited response [1], we obtain figure-of-merit ranging within $0.003-0.11 \mathrm{esu} \mathrm{cm} / \mathrm{s}$ range for DFWM at $579 \mathrm{~nm}$. By the order of magnitude it is close to $F M$ for $C_{60}$-fullerenes. We estimated for $C_{60}$ at $1064 \mathrm{~nm} F M \geqslant 0.03 \mathrm{esu} \mathrm{cm} / \mathrm{s}$ from the data [24], and 0.005 at $633 \mathrm{~nm}$ from [13]. Although the values of figure-of-merit for polydiacetylenes are considerably higher (we estimated $30 \mathrm{esu} \mathrm{cm} / \mathrm{s}$ for PDA/BMCU gel from the data [33]), the PIC J-aggregates seem to be an attractive molecular nonlinear medium.

The $\chi^{(3)}$ measured corresponds to low concentration aggregated dye solution (monomer concentration $c=5 \cdot 10^{-4} \mathrm{M}$ ). It is evident that nonlinearity is higher for concentrated solutions, since the lifetime as well as annihilation constant does not change considerably, at least until $c=10^{-2} \mathrm{M}$. Therefore it is possible to predict that the figure-ofmerit does not change much. The limit monomer density would be in molecular solid possessing aggregate properties. Langmuir-Blodgett technique is known to ensure $\mathrm{J}$-aggregation in monolayer, therefore, to estimate the possible highest nonlinearities, we extrapolated our data to molecular Langmuir-Blodgett solid. Following the assumption that the molecular solid containing J-aggregates would be characterised by a monomer packing density equal to that deduced from Langmuir-Blodgett room temperature isotherms, we normalised the $\chi^{(3)}$ value to the volume partition of active PIC molecules in solution.

The size of the PIC molecule in the solution was measured by taking the isotherms of the monomolecular layer produced on the water surface of a Langmuir-Blodgett trough. For the area of the PIC molecule we calculated $0.48 \mathrm{~nm}^{2}$. The height of the PIC molecule was estimated to $0.15 \mathrm{~nm}$ taking into account the length of the $\mathrm{C}-\mathrm{H}$ bond as well as the torsion angle of the PIC molecule $\left(50.6^{\circ}\right.$ in ethanol). The volume per molecule at closest packing amounts' to $0.072 \mathrm{~nm}^{2}$. The water molecules that are not active in mixing process take up an essential part of the volume. Therefore the active PIC molecules take only $2.25 \times 10^{-5}$ volume part of solution. Taking this into account, we estimate $(5 \pm 2) \cdot 10^{-8} \mathrm{esu} \mathrm{cm} / \mathrm{s}$ for third order non- 
linear susceptibility of molecular LB solid containing J-aggregate at $579 \mathrm{~nm}$. Although the above estimation is rather rough, these high values can be considered as an approach to estimate the nonlinear susceptibilities of bulk material under the situation of high dilution in solution.

The quantitative description of nonlinear four wave mixing in $\mathrm{J}$ aggregates needs further studies to separate real and imaginary parts of polarisation response, including not only single exciton resonances but those of multiply excited species, annihilation as well as secondary effects such as reversible aggregate disordering. In general, a better understanding of the nature of huge polarizibility of heterogeneous partially ordered molecular systems within and near exciton resonance is needed. We are in progress with further theoretical and experimental studies of the dispersion and intensity dependence of the DFWM in aggregates.

\section{CONCLUSION}

The dispersion curves of the DFWM efficiency have been discussed with respect to the absorption, optical density changes, and Kramers-Kronig relation. The dispersion of efficiency of converted signal intensity follows the maximal changes of the optical density more than the resonance conditions. The power law dependence of the converted signal is shown to have a minimum at exact resonance taking into account the exponent of the interacting intensities as an overall parameter. Annihilation and secondary vibrational excitation processes are responsible for the detected deviations from third order dependency in the resonance case. Secondary changes in absorption (refraction) observed within excitation pulse at high intensities provide an additional four wave interaction mechanism. Simultaneous action of electronic and secondary vibrational nonlinearities at high intensity results in a more complex dependence of DFWM signal on excitation intensity. The third order nonlinear susceptibility of thin PIC J-aggregate layers was estimated from the converted signal intensity normalised to the volume partition of active PIC molecules in solution to $5 \cdot 10^{-8}$ esu. 


\section{Acknowledgements}

The authors acknowledge financial support from the Thuringian Ministry of Science, Research and Culture. The experimental work was partly supported by the Deutsche Forschungsgemeinschaft within the Sonderforschungsbereich 196 "Physik und Chemie optischer Schichten". RG and AP acknowledge the International Science Foundation for partial support of equipment maintenance. Many valuable discussions with Prof. A. Piskarskas and Prof. A. Stabinis (Vilnius) are gratefully acknowledged.

\section{References}

[1] Gadonas, R., Feller, K.-H. and Pugzyls, A. (1994). Opt. Commun., 112, 157.

[2] Feller, K.-H., Gadonas, R., Krasauskas, V. and Pugzyls, A. (1993). Intern. Journ. Optoelectr., 8, 677.

[3] Ying Wang (1991). J. Opt. Soc. Am., B 8, 891.

[4] Spano, F. C. and Mukamel, S. (1989). Phys. Rev., A 40, 5783.

[5] Sasaki, F. and Kobayashi, Sh. (1993). Appl. Phys. Lett., 63, 2887.

[6] Ando, E., Miyazaki, J., Murimoto, K., Nakahara, H. and Fukuda, K. (1985). Thin Solid Films, 13, 21.

[7] Ishimoto, K., Tomimuro, H. and Seto, J. (1986). Appl. Phys. Lett., 49, 1677.

[8] Kawaguchi, T. and Iwata, K. (1988). Thin Solid Films, 165, 323.

[9] Yamada, T., Kajikawa, K., Ishikawa, K., Takezeo, H. and Fukuda, A. (1993). Thin Solid Films, 226, 173.

[10] Kafali, Z. H., Lindle, J. R., Pong, R. G. S., Bartoli, F. J., Lingg, L. J. and Milliken, J. (1992). Chem. Phys. Lett., 188, 492.

[11] Blau, W. J., Bryne, H. J., Cardin, D. J., Dennis, T. J., Hare, J. P., Kroto, H. W., Taylor, R. and Walton, D. R. M. (1991). Phys. Rev. Lett., 67, 1423.

[12] Gong, Q., Sun, Y., Xia, Z., Zou, Y. H., Gu, Z., Zhou, X. and Di Qiang (1992). J. Appl. Phys., 71, 2705.

[13] Rosker, M. J., Marcy, H. O., Chang, T. Y., Khoury, J. T., Hansen, K. and Whetten, R. L. (1992). Chem. Phys. Lett., 196, 427.

[14] Zhang, Z., Wang, D., Ye, P., Li, Y., Wu, P. and Wu, D. (1992). Opt. Lett., 17, 973.

[15] Kafali, Z. H., Bartoli, F. J., Lindle, J. R. and Pong, R. G. S. (1992). Phys. Rev. Lett., 68, 2705.

[16] Bogdanov, V. L., Viktorova, E. N., Kulia, S. V. and Spiro, A. S. (1991). Sov. Pisma JETP, 53, 100.

[17] Spano, F. C., Kuklinski, J. R. and Mukamel, S. (1990). Phys. Rev. Lett., 65, 211.

[18] Gagel, R., Gadonas, R. and Laubereau, A. (1994). Chem. Phys. Lett., 217, 228.

[19] Minoshima, K., Taiji, M., Misawa, K. and Kobayashi, T. (1994). Chem. Phys. Lett., 218, 67.

[20] Sundström, V., Gillbro, T., Gadonas, R. and Piskarskas, A. (1988). J. Chem. Phys., 89, 2754.

[21] Knoester, J. (1993). J. Chem. Phys., 99, 8466.

[22] Gadonas, R., Feller, K.-H., Pugzyls, A., Jonusauskas, G., Oberle, J. and Rulliere, C., J. Chem. Phys. (submitted).

[23] Gaizauskas, E., Feller, K.-H. and Gadonas, R. (1995). Opt. Comm., 118, 360.

[24] Flom, S. R., Pong, R. G. S., Bartoli, F. J. and Kafali, Z. H. (1992). Phys. Rev., B 46, 15598. 
[25] Pugzyls, A., Gadonas, R., Piskarskas, A. and Baltuska, A. (1995). Exp. Techn. Phys., 41, 57.

[26] Feller, K.-H., Gadonas, R. and Pugzyls, A., Adv. Mater. (submitted).

[27] Gaizauskas, E., Feller, K.-H. and Valkunas, L. (1996). Opt. and Quantum Electronics 28 (in press).

[28] Paillotin, G., Swenberg, C. E., Breton, J. and Geacintov, N. E. (1979). Biophys. J., 25, 513.

[29] Gadonas, R., Danielius, R., Piskarskas, A. and Rentsch, S. (1983). Bull. Acad. Sci. USSR Phys., 47, 151.

[30] Fidder, H., Knoester, J. and Wiersma, D. A. (1993). J. Chem. Phys., 98, 6564.

[31] Juzeliunas, G. Z. (1988). Phys. D-Atoms, Molecules and Clusters, 8, 379.

[32] Kobayashi, T. (1991). Nonlinear Optics, 1, 91.

[33] Carter, G. M. (1987). J. Opt. Soc. Am., B 4, 1018. 